\title{
Relative Efficacy of a Nutritional Intervention on Cognitive Performance Across the Adult Lifespan and During Early Cognitive Decline
}

\author{
Thomas B. Shea ${ }^{\mathrm{a}, *}$ and Ruth Remington ${ }^{\mathrm{b}}$ \\ ${ }^{a}$ Department of Biological Sciences, UMass Lowell, Lowell, MA, USA \\ ${ }^{\mathrm{b}}$ Department of Nursing, Framingham State University, Framingham, MA, USA
}

Accepted 30 May 2019

\begin{abstract}
A nutritional supplement has maintained or improved cognitive performance for healthy adults and individuals with mild cognitive impairment (MCI). Performance varied between 93 healthy adults aged 18-73 years versus 43 aged 75-85 years and among individuals with MCI. Healthy adult performance was stratified by age and for MCI as "intact" or "impaired" (Dementia Rating Scale guidelines). Some older individuals performed as well as younger individuals. All intact individuals with MCI maintained baseline performance; only impaired individuals receiving the supplement maintained baseline performance. Variation among elderly individuals can preclude observation of efficacy. Supplementation may maintain rather than improve performance for some individuals.
\end{abstract}

Keywords: Cognitive impairment, lifestyle modification, nutritional supplements, dementia

Lifestyle modifications including nutritional and social enrichments and cognitive exercise/training can enhance and preserve cognitive performance in older adults [1]. Moreover, multiple studies indicate that improved nutrition promotes and maintains cognitive performance throughout the adult lifespan [2-4]. Lifestyle modifications have the advantage that they can be initiated at any time, as opposed to the inherent compromise of waiting until sufficient cognitive decline has transpired to warrant diagnosis and prescription of pharmacological agents $[4,5]$. Moreover, individuals and/or their caregivers

\footnotetext{
${ }^{*}$ Correspondence to: Thomas B. Shea, Department of Biological Sciences, UMass Lowell, Lowell, MA 02154, USA. Tel.: +1 978934 2881; Fax: +1 978934 3044; E-mail: Thomas_Shea@umml.edu.
}

may remain unaware of early cognitive decline [6-9] and, if aware, may not seek assistance [10].

While non-pharmacological interventions can improve cognition and delay progression of mild cognitive impairment (MCI) to dementia, and in some cases may be as or more effective than pharmacological approaches, heterogeneity of subjects and variability in interventions and outcomes continued to limit firm conclusions [11-18]. Interpretation is further confounded with non-pharmacological approaches since participants are typically not naïve to treatments and participants may have inherent deficiencies in metabolism or differential absorptive capacity of nutrients [4]. Even with pharmacological interventions, combinatorial approaches display increased efficacy of both non-pharmacological and pharmacological therapies [4, 19]. 
A combinatorial nutritional supplement consisting of three vitamins (folic acid, B12, alpha-tocopherol) and three nutraceuticals (S-adenosyl methionine, $\mathrm{N}$-acetyl cysteine, acetyl-L-carnitine) maintained and improved cognitive performance for individuals with no known or suspected cognitive difficulties nor any other confounding illness (defined as "healthy" with respect to cognitive performance) [20], those diagnosed with MCI [21], and those diagnosed with Alzheimer's disease (AD) [22-24]. While overall improvement was observed in all of these studies, concerns were identified that warranted further investigation.

The first such concern was that healthy individuals $\leq 74$ years of age receiving treatment improved significantly in executive function while individuals >74-86 years of age ("elderly") did not. Several elderly individuals displayed extreme declines in performance during treatment, which prompted exclusion of the elderly cohort from conclusions regarding efficacy [24]. Recent analyses revealed that these participants in this elderly cohort declined in performance severely enough to be classified as outliers. It was concluded that these individuals were undergoing cognitive decline that did not as yet impair daily function but was revealed by cognitive testing [25]. Following their exclusion, performance of the treated elderly cohort did not differ significantly from the placebo cohort due to the remaining wide range of scores (resultant large standard deviation). However, the elderly cohort nevertheless demonstrated improvement comparable to the improvement of the younger cohort [25]. These latter findings prompted further comparison of the demographics and relative performance of all participants (young plus elderly) versus that previously presented for the young cohort only [21], which we have carried out herein.

The second concern was that participants with MCI statistically improved following treatment versus placebo on a cognitive test (the Dementia Rating Scale; DRS) that assesses performance across simple to complex domains but did not improve on a test that exclusively assesses executive function (the ClockDrawing Test; CDT) [21]. To examine this differential impact more closely, we compared herein the relative CDT performance of individuals demonstrating unimpaired versus impaired DRS performance.

Participants received the above combinatorial nutritional supplement as detailed previously [20-24]. Participants completed a number of tests at baseline and after receiving the formulation or indis- tinguishable placebo under double-blind conditions as follows:

The Trail-Making Test (TMT) assesses cognitive decline associated with progression of MCI, dementia and otherwise healthy aging [26]. Part A (TMT-A) calls for connection of a series of numbers in order. Part B (TMT-B) calls for connection of alternating numbers and letters in order. Performance is subject to age and education. Slowing of "switching" of tasks (i.e., alternating between numbers and letters) occurs across adult life [27]. Performance can reveal difficulties in neuromuscular coordination, following simple instructions (TMT-A) and executive function (TMTB) $[26,27]$. Executive function can be highlighted by subtracting an individual's score on TMT-A from TMT-B.

The Dementia Rating Scale (DRS) [29] assesses a variety of cognitive performance across a number of domains from simple to complex such as memory, attention, and conceptualization. As in our prior studies, we sorted individuals based on their performance on the DRS after 3 months of intervention or placebo as intact $(\geq 9)$, mildly impaired (6-8), moderately impaired $(4,5)$, and severely impaired $(\leq 3)$ as described in the DRS manual [28].

In the Clock-Drawing Test (CRT), which assesses executive function [29], participants are asked to draw the face of an analog clock, place the numbers within the face, draw a large and small hand (to indicate minutes and hours) with arrows on the hands, and to place the arrows to indicate a specified time (in this case, "12:45").

As previously shown, unlike individuals $\leq 74$ years of age, which were distributed equally according to age, gender, education, and baseline performance on the TMT, elderly individuals were distributed equally according to age, education, and gender but not according to baseline performance on the TMT following randomization (Table 1), which confounded interpretation [25]. Despite this difference within the elderly cohort, when we combined the elderly cohort (excluding the outliers as described in [25]), the total cohort was randomized in all respects (Table 1). In addition, the entire cohort displayed improvement following treatment versus placebo to an identical degree as that previously shown for the younger cohort alone (Table 1; see also [20]), and the elderly cohort displayed improvement to a statistically-identical degree as the younger cohort (Table 1). While this may be anticipated due to the larger number of individuals within the younger cohort (93 in the younger cohort versus 43 in the 
Table 1

Values present demographics and performance on the Trail-Making Test (*scores for part B - part A) at baseline and following 3 months of receiving the nutritional supplement (Treated) or Placebo for total participants and those participants stratified according to age

\begin{tabular}{|c|c|c|c|c|c|c|c|c|c|c|}
\hline & & \multicolumn{2}{|c|}{ Total Participants } & & \multicolumn{2}{|c|}{ Younger Participants } & & \multicolumn{2}{|c|}{ Older Participants } & \\
\hline & & Treated & Placebo & & Treated & Placebo & & Treated & Placebo & \\
\hline & $n$ & 72 & 64 & & 51 & 42 & & 21 & 22 & \\
\hline Gender & $\%$ Female & $32 \%$ & $31 \%$ & & $41 \%$ & $45 \%$ & & $48 \%$ & $47 \%$ & \\
\hline \multirow{2}{*}{ Age } & Range & $18-85$ & $18-86$ & & $18-73$ & $18-73$ & & $75-85$ & $75-86$ & \\
\hline & Mean (SD) & $66.9(12.9)$ & $68.4(12.0)$ & & $57.9(15.1)$ & $61.6(13.6)$ & & $78.9(2.9)$ & $79.1(3.6)$ & \\
\hline Education & $\% \geq$ College & 38 & 48 & & 43 & 31 & & 80 & 80 & \\
\hline TMT B-A* & & & & $p$ & & & $p$ & & & $p$ \\
\hline Baseline & Mean (SD) & $-0.4(27.1)$ & $3.1(24.0)$ & 0.5 & $2.6(28.8)$ & $4.7(20.7)$ & 0.7 & $2.6(26.9)$ & $-10.5(34.3)$ & 0.21 \\
\hline Post treatment & Mean (SD) & $-7.8(15.4)$ & $1.5(27.3)$ & 0.02 & $-7.4(14.4)$ & $3.1(29.5)$ & 0.04 & $-7.1(27.3)$ & $-15.8(33.9)$ & 0.35 \\
\hline
\end{tabular}

Data are from $[11,16]$. SD, standard deviation; $\geq$ College, completion of 4 years of college or more; $p, p$-values comparing Treated versus Placebo cohorts via 1-tailed Student's $t$ test.

older cohort after elimination of outliers), it nevertheless concealed the baseline differences among elderly individuals as well as the lack of statistical significance following treatment between elderly treated and placebo cohorts. Notably, the range of scores of treated individuals which did improve in the older cohort paralleled that of the younger cohort [20], indicating efficacy for some individuals. Separation of individuals according to ages was the only mechanism which revealed differences between younger and older cohorts.

To probe further the differential performance of individuals diagnosed with MCI on the DRS versus the CDT, these individuals were separated into cohorts demonstrating "intact" and "impaired" performance on the DRS. This classification revealed a unique, statistically-significant decline in performance for individuals in the impaired cohort receiving the placebo, while all others remained statistically identical to baseline values (Fig. 1).

These findings on healthy adults highlight the potential differential impact of nutritional intervention on cognitive performance among individuals and underscore that even those interventions that have significant improvement among cohorts with comparable age and baseline cognitive performance cannot be assumed to have a similar effect on each individual within any cohort. Comparison of differential statistical performance between younger versus older cohorts further demonstrates that the relative wider range of performance among elderly individuals, and the resultant wide standard deviation, can preclude observation of a significant difference in clinical studies, and, conversely, can mask relatively poorer performance of a subset of participants in a study encompassing diverse demographics.
These findings for individuals diagnosed with MCI parallel those previously observed for individuals with $\mathrm{AD}$ receiving the same nutritional intervention; individuals with $\mathrm{AD}$ demonstrating intact performance on the DRS also did not display improvement in the Clock-Drawing test versus placebo, while those with impaired DRS performance improved statistically versus their own baseline and versus those receiving placebo [21]. Similarly, individuals consuming this nutritional intervention for an extended period under open-label conditions did not display the decline characteristic of historical placebo cohorts $[23,24]$. MCI participants were close to the maximum possible score on the CDT (15 maximal points), leaving little room for improvement from baseline, but highlighting the significant decline for those classified as impaired according to the DRS. Analogous results were observed for participants diagnosed with

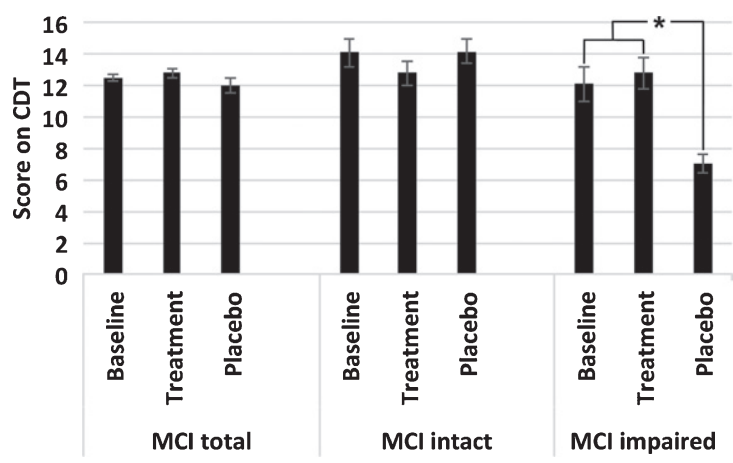

Fig. 1. Values represent the mean \pm standard error of performance at baseline and after receiving the nutritional supplement (Treatment) or placebo for 3 months on the Clock-drawing test for all participants with MCI (total) and those participants stratified as Intact or Impaired according to their respective performance on the DRS after 3 months of treatment or placebo. ${ }^{*} p<0.05$; Student's $t$ test. 
AD but classified as "intact" versus those classified as impaired according to the DRS [18]. These findings highlight that lifestyle modifications may in some cases not impart apparent improvement for individuals with initial high-level functioning but may nevertheless be involved in maintenance of existing levels of cognitive performance. These findings further highlight the advantage, and moreover the necessity, of using more than one cognitive test and, moreover, to employ tests that collectively encompass a range of cognitive performance from simple memory to executive function.

In these and prior analyses, we utilized only a singular lifestyle intervention-a combinatorial nutritional supplement. Of critical importance is to compare the efficacy of this and other nutritional interventions in combination with additional lifestyle modifications (e.g., increased social activity and mental and physical exercise) under similar conditions. Nevertheless, the current findings, taken together with the progressive decline in efficacy for cohorts displaying baseline impairment as shown herein and in prior studies [21-24], coupled with the identification of impaired performance of individuals who were unaware of any impairment [25], support initiation of lifestyle interventions as early as possible, including prior to any detectable cognitive impairment.

\section{ACKNOWLEDGMENTS}

The original acquisition of data referenced in these studies was supported by the Alzheimer's Association and by internal funds from UMass Lowell.

\section{CONFLICT OF INTEREST}

Dr. Shea is a non-salaried advisor for Sevo Nutraceuticals (Waltham, MA), which has licensed the formulation utilized in the above studies. Both Shea and UMass Lowell have a financial interest in this company and the formulation.

\section{REFERENCES}

[1] Kelly ME, Duff H, Kelly S, McHugh Power JE, Brennan S, Lawlor BA, Loughrey DG (2017) The impact of social activities, social networks, social support and social relationships on the cognitive functioning of healthy older adults: A systematic review. Syst Rev 6, 259.

[2] Dauncey MJ (2014) Nutrition, the brain and cognitive decline: Insights from epigenetics. Eur J Clin Nutr 68, 11791185.
[3] van de Rest O, Berendsen AA, Haveman-Nies A, de Groot LC (2015) Dietary patterns, cognitive decline, and dementia: A systematic review. Adv Nutr 6, 154-168.

[4] Shea TB, Remington R (2015) Nutritional supplementation for Alzheimer's disease. Curr Opin Psychiatry 28, 141-147.

[5] Koskas P, Pons-Peyneau C, Houenou-Quenum N, Romdhani M, Gasmi M, Galleron S, Drunat O (2018) Factors influencing time between onset of signs/symptoms and referral for dementia in elderly outpatients. Rev Neurol (Paris) 2174, 36-43.

[6] Bradford A, Kunik ME, Schultz P, Williams SP, Singh H (2009) Missed and delayed diagnosis of dementia in primary care: Prevalence and contributing factors. Alzheimer Dis Assoc Disord 23, 306-314.

[7] Barrett AM, Orange W, Keller M, Damgaard P, Swerdlow RH (2006) Short-term effect of dementia disclosure: How patients and families describe the diagnosis. J Am Geriatr Soc 54, 1968-1970.

[8] Jessen F (2014) Subjective and objective cognitive decline at the pre-dementia stage of Alzheimer's disease. Eur Arch Psychiatry Clin Neurosci 264, S3-S7.

[9] Vannini P, Amariglio R, Hanseeuw B, Johnson KA, McLaren DG, Chhatwal J, Pascual-Leone A, Rentz D, Sperling RA (2017) Memory self-awareness in the preclinical and prodromal stages of Alzheimer's disease. Neuropsychologia 99, 343-349.

[10] Begum A, Morgan C, Chiu CC, Tylee A, Stewart R (2012) Subjective memory impairment in older adults: Aetiology, salience and help seeking. Int J Geriatr Psychiatry 27, 612620 .

[11] Serrano-Pozo A, Growdon JH (2019) Is Alzheimer's disease risk modifiable? J Alzheimers Dis 67, 795-819.

[12] Horr T, Messinger-Rapport B, Pillai JA (2015) Systematic review of strengths and limitations of randomized controlled trials for non-pharmacological interventions in mild cognitive impairment: Focus on Alzheimer's disease. J Nutr Health Aging 19, 141-153.

[13] Lin JS, O'Connor E, Rossom RC, Perdue LA, Burda BU, Thompson M, Eckstrom E (2013) Screening for Cognitive Impairment in Older Adults: An Evidence Update for the U.S. Preventive Services Task Force [Internet]. U.S. Preventive Services Task Force Evidence Syntheses, formerly Systematic Evidence Reviews, Agency for Healthcare Research and Quality (US), Rockville (MD). Report No. 14-05198-EF-1.

[14] Fenech M (2017) Vitamins associated with brain aging, mild cognitive impairment, and Alzheimer disease: Biomarkers, epidemiological and experimental evidence, plausible mechanisms, and knowledge gaps. Adv Nutr $\mathbf{8}$, 958-970.

[15] Liang JH, Xu Y, Lin L, Jia RX, Zhang HB, Hang L (2018) Comparison of multiple interventions for older adults with Alzheimer disease or mild cognitive impairment: A PRISMA-compliant network meta-analysis. Medicine (Baltimore) 97, e10744.

[16] Sherman DS, Mauser J, Nuno M, Sherzai D (2017) The efficacy of cognitive intervention in mild cognitive impairment (MCI): A meta-analysis of outcomes on neuropsychological measures. Neuropsychol Rev 27, 440-484.

[17] McMaster M, Kim S, Clare L, Torres SJ, D'Este C, Anstey KJ (2018) Body, Brain, Life for Cognitive Decline (BBL$\mathrm{CD}$ ): Protocol for a multidomain dementia risk reduction randomized controlled trial for subjective cognitive decline and mild cognitive impairment. Clin Interv Aging 13, 2397 2406. 
[18] Botchway BOA, Moore MK, Akinleye FO, Iyer IC, Fang M (2018) Nutrition: Review on the possible treatment for Alzheimer's disease. J Alzheimers Dis 61, 867-883.

[19] Cummings JL, Tong G, Ballard C (2019) Treatment combinations for Alzheimer's disease: Current and future pharmacotherapy options. J Alzheimers Dis 67, 779-794.

[20] Chan A, Lepore A, Kotoya E, Zemianek J, Remington R, Shea TB (2010) Efficacy of a vitamin/nutriceutical formulation on cognitive speed and recall in adults with no known or suspected dementia. J Nutr Health Aging 14, 224-230.

[21] Remington R, Lortie J, Hoffman H, Page R, Morrell C, Shea TB (2015) A nutritional formulation for cognitive performance in mild cognitive impairment: A placebo-controlled trial with an open-label extension. J Alzheimers Dis 48, 591-595.

[22] Remington R, Bechtel C, Larsen D, Lortie JJ, Samar A, Doshanjh L, Fishman P, Luo Y, Smyers K, Hoffmann H, Barysauskas C, Barton B, Shea TB (2015) A nutritional formulation for cognitive performance and mood in Alzheimer's disease: A phase II multi-site randomized trial with an open-label extension J Alzheimers Dis 45, 395-405.

[23] Remington R, Bechtel C, Larsen D, Samar A, Page R, Morrell C, Shea TB (2016) Maintenance of cognitive performance and mood for individuals with Alzheimer's disease following consumption of a nutraceutical formulation: A one-year, open-label study. J Alzheimers Dis 51, 991-995.
[24] Chan A, Paskavitz J, Remington R, Shea TB (2008) Efficacy of a vitamin/nutriceutical formulation for early-stage Alzheimer's disease: A one-year open-label pilot study with a 16-month extension. Am J Alzheimers Dis Other Demen 23, 571-585.

[25] Shea TB, Remington R (2018) Apparent cognitive decline as revealed by an executive function test within a cohort of elderly individuals self-reporting normal cognitive performance. J Alzheimers Dis 61, 913-915.

[26] Bowie CR, Harvey PD (2006) Administration and interpretation of the Trail Making Test. Nat Protoc 1, 2277-2281.

[27] Connor KO, Chaisson C, Green RC, Stern RA (2008) Trail Making Test errors in normal aging, mild cognitive impairment, and dementia. Arch Clin Neuropsychol 23, 129-137.

[28] Jurica, PJ, Leitten, CL, Mattis S (2001) DRS-2 Dementia Rating Scale Professional Manual. PAR, Lutz, FL.

[29] Royall DR, Cordes JA, Polk M (1998) CLOX: An executive clock drawing test. J Neurol Neurosurg Psychiatry 64, 588594. 